\title{
L'ASTRONOMIE DES ETOILES DOUBLES FACE A LA MISSION ASTROMETRIQUE DU SATELLITE HIPPARCOS
}

J. DOMMANGET

Observatoire Royal de Belgique

(Communications Observatoire Royal de Belgique, Serie B, No 127)

SUMMARY :

The HIPPARCOS satellite is described as well as the scanning process of the sky by its optical design, as a consequence of the satellite's motion. From these data, the possibilities of the mission is deduced and applied in the case of the binaries.

Discussion is made of what may be expected for different double star categories including spectroscopic, astrometric, close visual and wide pairs. Information are given on how proposals should be made for lists of binaries that should be observed.

\section{DISCUSSION}

KREIDL: The plans should stand for Hipparcos to be reactivated after the initial 2.5 year survey. After a pause of ten years or so, redoing all measurements would provide a wealth of new information on proper motions and other quantities.

STRAND: What is the status of the Hipparcos project? Has it reached the feasibility or engineering phase?

DOMMANGET: In March 1980 ESA approved the project, and it is now in what is known as the phase B (announcement of opportunity to the industries) stage.

BAUM: An important scientific reason for keeping Hipparcos in operation much longer than the 2.5 years (or switching it back on from time to time in future decades) is its ability to test a large number of nearby stars for the presence of planetary systems. Space Telescope will have to limit its attention to a relatively few such candidates. The discovery of extra-solar planetary systems would have a terribly important impact on the future of space science.

RAKOS: I would like to add that the project has been approved and is going on with highest priority, but there is a big lack in the reduction program. We just do not know how to use this big amount of data; there are difficulties, but nobody knows how to solve them.

DOMMANGET: If it appears possible to introduce binaries - these trouble stars - in the observation program, a first reduction will be done by rejecting all close binaries to establish the main astrometric catalog. Only afterward will it be possible to consider information about the binaries for particular reductions. 